\title{
Hossein Jadvar, Heather Jacene and Michael Graham. Molecular Imaging: An Introduction
}

\author{
Cambridge University Press, Cambridge (UK), 2017 ISBN: 978-1-62,623-248-8
}

\author{
Giuseppe Danilo di Stasio ${ }^{1} \cdot$ Luigi Mansi ${ }^{2}$
}

Published online: 28 March 2018

(C) Springer-Verlag GmbH Germany, part of Springer Nature 2018

This book is edited by Hossein Jadvar, past president of American College of Nuclear Medicine (ACNM) and Society of Nuclear Medicine and Molecular Imaging (SNMMI), and a member of the faculty at the Keck School of Medicine, University of Southern California, Los Angeles; Heather Jacene, from the Harvard Medical School in Boston; and Michael Graham, University of Iowa, Iowa City.

This little volume of 82 pages aims to provide up-todate information to introduce the reader to the everchanging subject that is molecular imaging. Thus, it could be useful for students, physicians in training and other professionals who want to approach the basic concepts of molecular imaging in a direct manner and in a relatively short length of time.

The title of the book, Molecular Imaging: An Introduction, is self-explanatory with respect to its purpose. The editorial keywords are simplicity and synthesis. In less than 90 pages, the publication is organized into 15 chapters, which ideally divide the volume into two sections. The first section provides the reader with a concise introduction to instrumentation, physics and methods of various imaging modalities, which include Computed Tomography (CT) and Magnetic Resonance Imaging/Spectroscopy (MRI/MRS), optical and ultrasound imaging, and nuclear medicine, with Single Photon Emission Computed Tomography (SPECT) and Positron Emission Tomography (PET). The book continues with an evaluation of several key biological processes which may be included in the scenario of interest for molecular imaging. In particular, traditional issues such as perfusion, metabolism, cellular proliferation, hypoxia, angiogenesis and apoptosis are treated together with more complex environments that molecular imaging can analyze, such as reporter genes, stem cell tracking and amyloid imaging. Interestingly, each chapter presents at its end a quick reference bibliography to which the reader may refer to deepen their understanding of a specific topic. Therefore, the book offers a new and refreshing introduction to molecular imaging, aided by the inclusion of a varied collection of figures and diagrams, which are present in each of the 15 chapters, with the exception of the third chapter, effectively highlighting the major concepts for the reader. This clearly immense and complex work is explained in a simple and discursive way, all within a relatively small number of pages, dense but easy to read.

Molecular imaging is a relatively new field of study that provides valuable information to improve patient diagnosis at all levels. This book represents a valuable instrument which should be especially helpful to nuclear medicine and radiology residents, but may also be useful to both undergraduate and postgraduate students who wish to seize the basic concepts of molecular imaging in a direct and concise manner. Clearly, it could also represent an important cultural instrument for all the physicians and other professionals who want to familiarize themselves with this new diagnostic scenario.

Luigi Mansi

mansi.luigi@libero.it

University Federico II, Napoli, Italy

2 Interuniversity Research Center for Sustainability (CIRPS), Napoli, Roma, Italy 